\title{
A Study on the Size of Tangible Organ-shaped Controllers for Exploring Medical Data in VR
}

\author{
Anke V. Reinschluessel \\ University of Bremen \\ Digital Media Lab \\ Bremen, Germany \\ areinsch@uni-bremen.de \\ Verena Uslar \\ University Hospital for Visceral \\ Surgery, University of Oldenburg \\ Oldenburg, Germany
}

\author{
Thomas Muender \\ University of Bremen \\ Digital Media Lab \\ Bremen, Germany \\ thom@uni-bremen.de \\ Thomas Lück \\ cirp $\mathrm{GmbH}$ \\ Heimsheim, Germany
}

\author{
Tanja Döring \\ University of Bremen \\ Digital Media Lab \\ Bremen, Germany \\ tanja.doering@uni-bremen.de \\ Dirk Weyhe \\ University Hospital for Visceral \\ Surgery, University of Oldenburg \\ Oldenburg, Germany
}

\author{
Andrea Schenk \\ Fraunhofer Institute for Digital \\ Medicine MEVIS \\ Bremen, Germany
}

\author{
Rainer Malaka \\ University of Bremen \\ Digital Media Lab \\ Bremen, Germany
}

\begin{abstract}
Virtual reality (VR) is, by nature, excellent in showing spatial relationships, e.g. for viewing medical 3D data. In this work, we propose a VR system to view and manipulate medical 3D images of livers in combination with $3 \mathrm{D}$ printed liver models as controllers. We investigate whether users benefit from a controller in the shape of a liver and if the size matters by using three different sizes (50\%, $75 \%, 100 \%$ ). In a user study with 14 surgeons, we focused on presence, workload and qualitative feedback such as preference. While neither size differences nor the VIVE tracker as control resulted in significant differences, most surgeons preferred the $75 \%$ model. Qualitative results indicate that high similarity of physical and virtual objects regarding shape and a focus on good manageability of the physical object is more important than providing an exact replica in size.
\end{abstract}

\section{CCS CONCEPTS}

- Human-centered computing $\rightarrow$ Virtual reality; Haptic devices; User studies; • Applied computing $\rightarrow$ Health care information systems; $\bullet$ Hardware $\rightarrow$ Haptic devices.

\section{KEYWORDS}

Medical Imaging; Image Navigation; Spatial Interaction; Virtual Reality; VR; 3D printing; 3D Model; User Study; Interview; Surgery

ACM Reference Format:

Anke V. Reinschluessel, Thomas Muender, Tanja Döring, Verena Uslar, Thomas Lück, Dirk Weyhe, Andrea Schenk, and Rainer Malaka. 2021. A

\section{(c) (i) (8)}

This work is licensed under a Creative Commons Attribution-NonCommercial International 4.0 License.

CHI '21 Extended Abstracts, May 8-13, 2021, Yokohama, Japan

(C) 2021 Copyright held by the owner/author(s)

ACM ISBN 978-1-4503-8095-9/21/05.

https://doi.org/10.1145/3411763.3451594
Study on the Size of Tangible Organ-shaped Controllers for Exploring Medical Data in VR. In CHI Conference on Human Factors in Computing Systems Extended Abstracts (CHI '21 Extended Abstracts), May 8-13, 2021, Yokohama, Japan. ACM, New York, NY, USA, 7 pages. https://doi.org/10. $1145 / 3411763.3451594$

\section{INTRODUCTION}

Virtual reality (VR) is by nature excellent in showing spatial relationships through its stereoscopic displays. In medicine, especially when it comes to surgery involving cancer resection, spatial relationships of blood vessels and tumour tissue are very important. And as the distances between tumour tissue and blood vessels are not easy to determine from routine 2D MRI (magnetic resonance imaging) and CT (computed tomography) data, 3D models come in handy - which can be created based on this data (for the process of model reconstruction cf. [15, 19, 22]). Despite efforts to create automatic 3D visualisations from $2 \mathrm{D}$ images (e.g. [27, 28]), to the best of our knowledge, it is still standard to view the routine 2D images as created by CT and MR scanners (although this might vary depending on the country) [6]. In most cases, physicians have to create their own mental 3D model based on the images and their prior anatomical knowledge. This is challenging and as a cooperating head surgeon stated "The ability to imagine the 3D situation can decide between a palliative or a curative approach for the patient." Research by Morineau et al. [17] suggests that this surgical planning and decision making depends on the level of expertise. The aforementioned 3D models can support the surgeon in this decision process by showing a more comprehensive view of the spatial relationships. The interaction with 3D visualisations is challenging, starting with the rudimental tasks like viewing and manipulation, there have been efforts to make it easier and tangible [3]. For example, Reitinger et al. [21] suggested using a special input device or Faludi et al. [5] investigated using force fields in combination with VR. Instead of viewing the respective models in VR, one could print them three-dimensionally (3D). With the rise of 3D printing, also 
the application in the medical domain has increased within the last 20 years [24]. A literature review by Martelli et al. [16] shows the variety of application scenarios and its benefits. Yet, 3D prints (a) do not allow for manipulating the viewed content (e.g., changing colour schemes, omitting structures etc.), (b) are fixed in size and (c) have problems regarding light refraction of the materials. However, their shape matches the real organ and as performing surgeries is a manual task, the haptic and "tangible" properties can be of advantage. Research from anatomy learning shows that being able to experience the anatomy first hand increases the learning outcome [11] and therefore presenting the information with a realistic physical shape might also have a positive impact on the surgery results.

Research by Kwon et al. [13] done in playful augmented reality setups can be interpreted in a way that participants perform best with virtually and physically equally shaped and sized objects. As they could not find any effects of the size alone, the authors argued that regarding size more research is needed. Simeone et al. [23] found that for playful VR environments physical props should have the same shape and should not differ too much from their virtual representation. Therefore, to the best of our knowledge, until now, if controllers were designed for VR, the strategy was either matching in size or neglecting this aspect as in the work by Hinckley et al. [8].

In this work, we propose a prototypical system that uses 3D prints in the shape of the respective organ in combination with VR. Research on tangible user interfaces (TUIs), e.g. created via 3D printing, suggests that having an interaction device in the object's real shape results in higher satisfaction $[1,12,18]$ and that tangible interaction is faster and more intuitive [2]. A possible explanation for these effects is that it is benefiting from the natural spatial memory of humans [4]. TUIs build on the users' knowledge and skills of interaction with the real, non-digital world and therefore are often associated with reality-based interaction [10]. In particular, TUIs take advantage of natural physical affordances to achieve an increased legibility and seamlessness of interaction between people and digital information [9].

Following up on our previous research [19, 20], we focus on the liver as a use case in order to get a first scientifically proven hypothesis on the optimal size of a liver-shaped controller. That size is a relevant design decision was just shown by López García and Hornecker [14] in the context of data physicalisation. The liver is a challenging structure, as it is a large organ with a transversal size of $20-23 \mathrm{~cm}$ (7.87-9.05 inch), a lateral size of $15-18 \mathrm{~cm}$ (5.90-7.08 inch) and a weight of roughly $1400-1800$ gram (49.4-63.5 oz) ${ }^{1}$, depending on gender, age, body size, weight and health. Therefore, this work investigates whether the controller has to be of the same dimensions as the original organ shown in VR. Moreover, our research addresses if a liver-shaped tangible controller reduces the mental workload for the user while manipulating the VR display. Thereby, this work presents a novel but preliminary take on the interaction in the medical domain with medical 3D models and how an intuitive interaction device, i.e. controller, could look like. Our study reveals that the matching in size is not as important as being able to handle the object in size and especially weight. Nevertheless, a match in shape seemed to inspire specific types of gestures related to the

\footnotetext{
${ }^{1}$ https://radiopaedia.org/articles/hepatomegaly
}

medical background and the organ itself. Yet, our results indicate that further investigations are needed to explicitly focus on the weight while evaluating smaller size changes against each other.

\section{METHOD}

To investigate whether physicians see a potential for tangible interaction to explore organ models and to determine which size they prefer, we implemented a VR prototype with four different tangible controllers for interaction: three differently sized objects in the shape of a liver and one standard VIVE Tracker.

\subsection{VR Prototype}

The prototype was developed in the Unity $2018.4^{2}$ game engine in combination with a VIVE Pro $^{3}$ head mounted display (HMD). The virtual scene consisted of a white room and one of four different virtual organ models, see Figure 1. The virtual models were created based on anonymised MR images of real patients of a local hospital. All patients gave informed consent to use their data for this purpose. The images were annotated by medical technical assistants using dedicated planning software and combined to a polygonal model [22]. The virtual model was linked to the same location as the tangible based on the results by Ware and Rose [25] and calibrated to match the 3D prints of the liver in orientation. The virtual model was always displayed in the size of the real liver $(100 \%)$. The only possible interaction was viewing and turning the model by using the tangibles described in the next section.

\subsection{Tangibles}

To control the virtual liver models in VR, three tangibles were created, representing the outside shape of a healthy liver. All three models have the same shape but different sizes of $100 \%(25 \mathrm{~cm}$ (9.84 inch), 1072 gram $\left.(37.81 \mathrm{oz})^{4}\right), 75 \%$ (19 cm (7.48 inch), 594 gram $(20.95 \mathrm{oz}))$, and $50 \%$ (12.5 cm (4.92 inch), 241 gram ( $8.5 \mathrm{oz}))$ of the real liver size, see Figure 2. We chose these three sizes because of the following reasons: Choosing a size larger than $100 \%$ would have been unreasonable because livers are quite large $(100 \%=$ $25 \mathrm{~cm}$ (9.84 inch)) and therefore handling is already hard as seen in qualitative feedback and in prior informal testing for the study design. Previous research revealed early stage results that a similar size is good [13] but it was always evaluated with smaller objects that are easy to hold in one hand. Evaluating previous 3D-printed liver models under different aspects showed that the $50 \%$ liver fits the conditions for "fitting the hand well". As we wanted to evaluate at least one increment in between, we also added a model in the size of $75 \%$. These three different sizes plus one baseline condition present a good trade-off in terms of ecological aspects, number of conditions and time constraints. The models were created by $3 \mathrm{D}$ printing with the laser sintering technique ${ }^{5}$. The material used is a specific polymer (PA12), typically used for this type of 3D printing. With this printing technique the production costs are between $100 €$ (approx. \$114, small model) and up to $500 €$ (approx. $\$ 569$, large model). The initial printing color is white but two of

\footnotetext{
${ }^{2}$ https://unity3d.com

${ }^{3}$ https://www.vive.com/us/product/vive-pro/

${ }^{4}$ All weights include the mount for the VIVE Tracker and the tracker itself for tracking.

${ }^{5} \mathrm{https}$ ://www.me.utexas.edu/images/NAMRAC_1987.pdf
} 

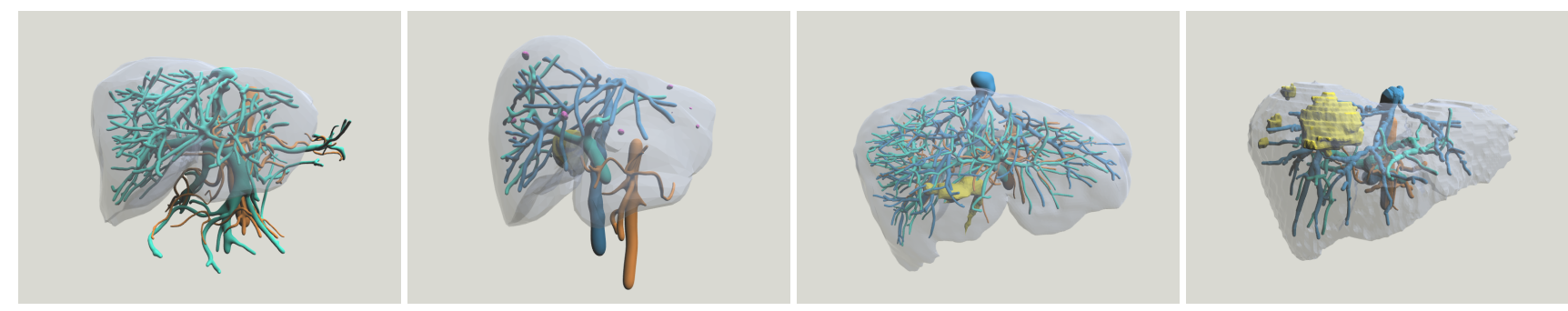

Figure 1: The virtual models used in the VR application. For all models the same color scheme was used (best seen in colour): orange - hepatic artery; blue - hepatic vein; cyan - portal vein; yellow - tumour; magenta - other lesions.
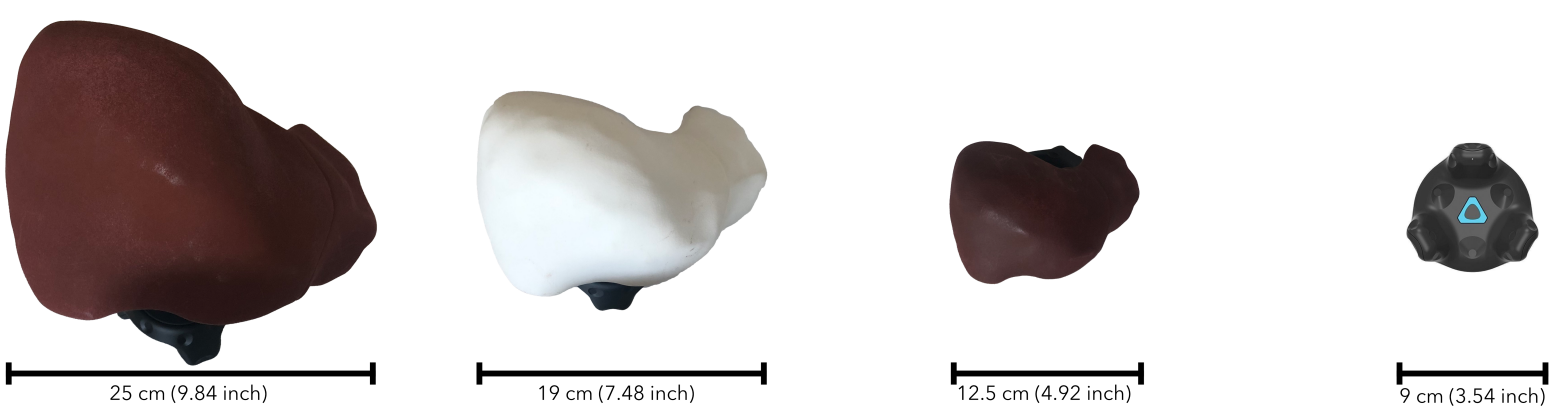

Figure 2: The tangible organ models - from left to right: Large - $100 \%$, Medium - $75 \%$, Small - $50 \%$. Scales are relative to the real patients' organ size. For tracking a VIVE Tracker was attached to the back of every tangible.

the models were painted in a more realistic color of the liver for illustrative purpose ${ }^{6}$.

\subsection{User Study}

We ran a user study with fourteen (14) participants (7 female, 7 male) to evaluate the sizes of our tangibles. The participants were recruited at a local university hospital using purposive sampling. Besides three medical students, resp. medical interns, (one of them was an educated surgery nurse), all participants had experience in general and/or visceral surgery. The participants were between 23 and 54 years old (mean $=36.9$ years), had professional experience in their field from 0.5 to 20 years $($ mean $=7.9$ years, median $=5.75$ years, $\mathrm{SD}=7.13$ ) and had performed a total number of $\sim 208$ liver surgeries as lead surgeon $($ mean $=15, \mathrm{SD}=28.5)$ and $\sim 336$ liver surgeries as assistant surgeon (mean $=24, \mathrm{SD}=35.4$ ).

After giving informed consent, participants were assigned to one of the four conditions (tracker, small 3D print (50\%), medium 3D print $(75 \%)$, large 3D-print $(100 \%)$ ) to start with. The order of conditions and virtual 3D models was pseudo-randomized across participants using a latin-square scheme to counterbalance for potential biasing effects. The participants performed all four conditions and filled out a questionnaire after each condition. Each condition consisted of two types of tasks: First, they had to answer two questions, which relied on their medical expertise and where specific for each virtual model. The questions were for example "In which segment

${ }^{6}$ The differences in color have no impact on the user study as participants could not see the tangibles before and during the study. is the tumor located?". The questions' primary goal was to make the participants turn and explore the models. The answers were not part of the evaluation itself as the performance highly depended on the background of the participants, their specific training and recollection of anatomical knowledge and therefore would not have been comparable.

Second, the participants were instructed to rotate the model into a given target orientation. An uncolored version of the virtual organ was displayed $40 \mathrm{~cm}$ (15.74 inch) in front of the user to view the target rotation. The model was rotated randomly on all three axes within given constraints to control the difficulty. Three rotation tasks with increasing complexity were performed. The task completion time as well as rotational error was recorded.

The questionnaire consisted of the Nasa TLX questionnaire [7] and the presence questionnaire by Witmer and Singer [26] (revised version by the UQO Cyberpsychology Lab, 2004) as it includes items that address involvement and sensory factors and has been used in a large number of studies. After all conditions, we collected basic demographic information (age, expertise level, hand size, number of performed liver surgeries and experience with VR and 3D visualizations) and performed a structured interview. We asked which model they preferred and about their reasons for the preference, how they perceived the size difference of the VR model and the physical model, and their opinion on integrating the proposed system into their daily workflow. The procedure took approximately 30 minutes. The participants did not receive any compensation for their participation. 


\section{RESULTS}

Table 1 shows the interview results about the device preference. The first column depicts how many participants preferred which device (three participants could not decide between two sizes so that both of their favorite controllers were included). Overall, the $75 \%$ liver was liked most: eight participants explained their favour for the medium-sized controller. They found it had the best size to handle and a good weight. The small and large livers were each preferred by four participants: the rationale for choosing the small liver was also because of its size, but also due the shape; the participants deciding for the large liver liked that it resembled the original size of the organ. One participant voted for the tracker because $s /$ he found it easier to handle due to the small size.

\begin{tabular}{lcc} 
Controller & Most liked & Least liked \\
\hline Tracker & 1 & $\mathbf{5}$ \\
Small liver (50\%) & 4 & 4 \\
Medium liver (75\%) & $\mathbf{8}$ & 1 \\
Large liver (100\%) & 4 & 4 \\
\hline
\end{tabular}

Table 1: The preferences and dislikes of the participants for each controller option (multiple answers were possible).

Asked about their least favourite controller option the participants responded with the distribution shown in the second column of Table 1 (multiple answers were possible). The reason given for the tracker (five votes) were primarily the shape, which they did not like while interacting with it. One participant stated that the tracker "stresses me most". The participant's objection regarding the small model (four votes) was its size in terms of being too small and a too large mismatch between what they feel and what they see in VR (three participants). The medium model was rejected by one participant because of its weight. The reasons given for liking the large model the least (4 votes) were primarily its weight (1072 gram (37.81 oz)) but also the size. It was mentioned that the handling was cumbersome and that the participants worried about dropping it.

Figure 3 shows a scatter plot of the hand sizes - split into hand width and finger length (both in $\mathrm{cm}$ ) - and the preferred model (by colour). Due to the participants' limited availability only data from 11 out of 14 participants could be gathered and are depicted here. The results indicate, that the participants preferring the $50 \%$ model all have a smaller hand width than the ones preferring the $100 \%$ model.

As only the large liver had the same size as the models in VR, we asked about if and how the participants perceived this mismatch. Four participants stated explicitly that they noticed the mismatch of size. It was especially noticeable with the small model and one said that $\mathrm{s} /$ he experienced it particularly for the right lobe of the liver. The remaining participants either answered that they somehow noticed it but did not care about it as they were primarily focusing on the VR visualisation or even did not recognise it explicitly. During the interviews we noticed that some participants mistook the medium liver for the real size liver model (which would be $100 \%$ as in the large one).

Furthermore, we asked for their opinion about possible benefits, drawbacks and integration in the daily workflow. All participants saw value in the proposed system and 13 out of 14 agreed that having a similar shaped device is beneficial for interaction, because it results in a better experience and supports visual thinking. The most mentioned application, which could benefit, was preoperative planning of liver surgeries, but also pancreas surgery, adrenal glans tumour resection and lung surgery. Additionally, education areas like anatomy learning and patient education were recommended. Observations during the study showed that participants counted liver segments - either in the air or on the model itself -, when the tasks asked for a specific liver segment, but only with the liver shaped models (not with the standard controller). They did this independent of the model's size as it was observed for all three sizes of the $3 \mathrm{D}$ printed liver controller.

The individual results for the presence subscales are shown in Figure 4. A Friedman-test on the presence subscales revealed significant differences between conditions for realism $\left(\chi^{2}=11.46\right.$, $p=.0094)$. Yet, a post-hoc pairwise comparison for the realism subscale using Holm-corrected Wilcoxon-tests did not reach significance. The results for the NASA TLX questionnaire are shown in Figure 5, lower values are better except for the performance subscale. The statistical analysis (Friedman-test) revealed no significant differences. For the rotation task a linear regression between accuracy (rotation error) and task completion time was done and the results were not significant. Therefore, we performed a Friedmantest, which also did not reach significance. We omit the results to increase readability and attach them as supplementary material.

\section{DISCUSSION}

The qualitative results from our interviews show a clear preference for the 3D printed models over the control group (VIVE Tracker) and especially a strong preference for the medium sized (75\%) liver model (8 vs. 4 for each of the other two models). Participants even thought, according to their utterances, that they were handed the real size model, when actually using the $75 \%$ model. This might indicate that it is not always necessary to build real-sized tangibles for interaction and reduced versions might have the same or even better effects - as long as they are not too small. Nevertheless, this clear preference can not be found in the quantitative data as neither the results from the presence and task load questionnaires nor the performance measures from the rotation task show significant differences. However, the median in the "mental demand" subscale indicates that the smallest model led to higher mental demand compared to all other conditions. These non-significant results might originate from too similar tasks and the fact that the participants focused more on the virtual representation, which was more interesting for them from a medical perspective. Some participants mentioned focusing more on the virtual model. Additionally, the virtual environment was empty and the same besides the virtual 3D model and therefore it is unsurprising that there were not any changes to presence. The subscale "performance" from the NASA TLX shows the highest median for the medium sized model, although the variance is the highest among all models. The high variance of this subscale in general could be due to the fact that only this scale is inverted on the questionnaire itself, thus leading to inverted answers. 


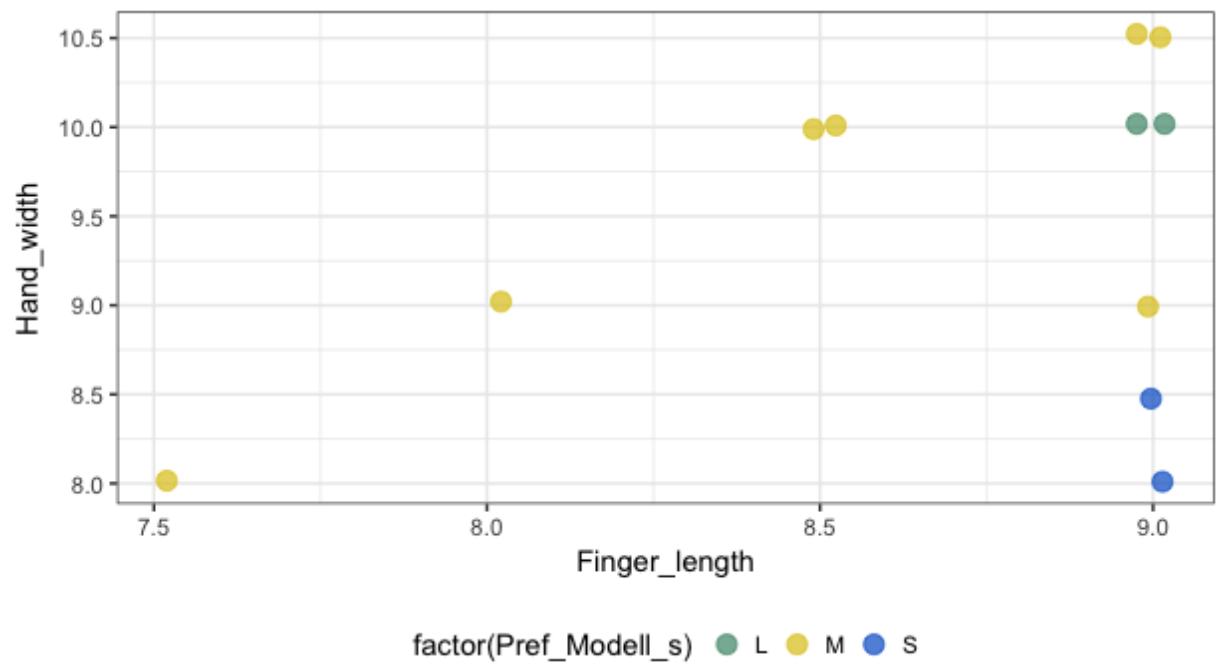

Figure 3: Scatter plot of hand width (in cm), finger length (in $\mathrm{cm}$ ) and chosen preferred model (by colour).

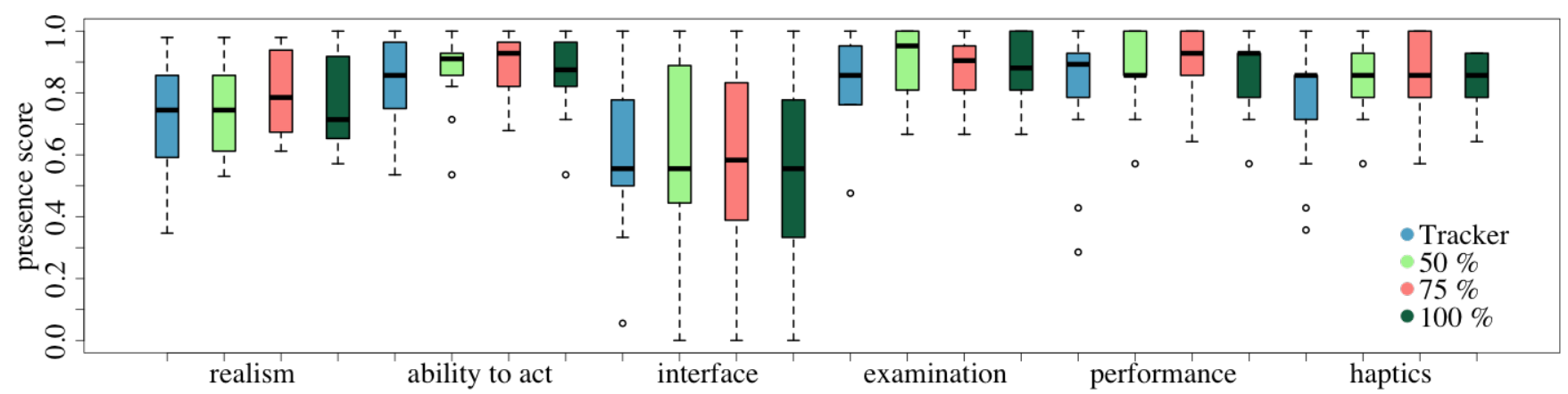

Figure 4: Boxplots (including median values) showing the results (the higher the better) of the presence questionnaire subscales: realism, ability to act, interface, examination, performance, and haptics.

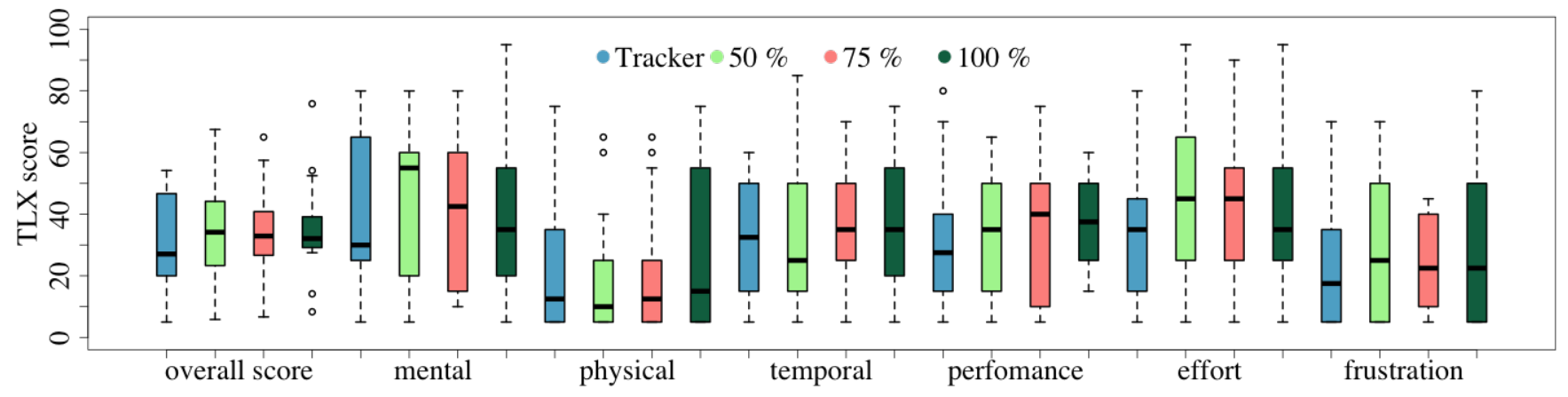

Figure 5: Boxplots (including median values) showing the result of the Nasa TLX questionnaire including the subscales: mental (demand), physical (demand), temporal (demand), performance, effort and frustration. 
In line with earlier studies that have addressed varying shapes or sizes of tangibles as physical props for virtual or augmented reality $[13,23]$, we found that the physical shape should be quite similar to the virtual counterpart while at the same time the users are more tolerant with the size of the physical object. In general, it seems worthwhile to be close to the virtual size, but if the task affords a manipulation of the object, potentially with additional selection or pointing, there is a trade-off to face between presenting the physical object in the real size and picking a size that fits well to the size of the hands and is comfortable to manipulate. In contrast to these earlier studies, which took place in simple test environments or playful arrangements, our use case presents an expert environment, in which the objects are potentially used for surgery planning and thus are very important in every aspect of their presentation. In this regard, our study presents novel and very useful insights: even if a realistic size of the physical presentation is possible, the majority of experts prefer a slightly smaller but in its size better manageable real object.

Overall the $75 \%$ model was preferred over various hand sizes, yet the hand size seems to have an influence on the choice. The plot in Figure 3 shows how smaller hand widths can result in a preference for smaller models while the participants preferring the larger model all had wider hands. The weight of the models could also have an effect, as the large model was quite heavy and needed some effort to hold up and interact with. This is also shown as a trend in the subscale "physical demand" of the task load questionnaire, which has the highest value for the large model. Similar results were found by [12] indicating that tangibles with more than $1 \mathrm{~kg}$ (2.2 pounds) are not well suitable for interaction.

As in future iterations of the prototype further interactions are planned, which would require the user to hold the model in one hand and interact with the other, comfortable manageability of the physical object becomes even more important. Possible interactions include annotations such as highlighting abnormal anatomical structures or marking resection planes for tumour removal and change of views, like just showing veins or just arteries and scaling the virtual model to get a better view. These tasks further support smaller models that fit the hands compared to the $100 \%$ model, which was described as unhandy and was generally held with both hands.

The weight was also mentioned by the participants as a factor to reject the large model. This might be a confounding factor, but with the large/100\% model we aimed at a more realistic experience, which could also include a more typical weight (a liver weights between 1400-1800 gram (49.4-63.5 oz)). Additionally, we used the same workflow and same printing technology to create all three models in order to have a comparable workload regarding preparation resulting in the given weight of the models. Furthermore, in the previous workshops creating patient individual models was discussed as one option, which would call for a quick production cycle as needed before the actual surgery. Yet, this presents valuable insights towards the future development of interaction devices of this size: for future evaluations different variants of creating the models should be incorporated, to test if the large model would have a higher acceptance rate with less weight and maybe even the medium one, as the same feedback was occasionally mentioned. Additionally, as the presence did not seem to be influenced significantly by the models, we will omit such measures when using a plain virtual environment with a focus on a single element in the scene in the future to shorten study time for the participants or substituting it with a more meaningful measurement.

Despite the mentioned issues regarding weight nearly all participants saw benefits in using a $3 \mathrm{D}$ printed organ model to interact with the related medical images. The observation that they counted liver segments aligns well with their self-reported supported visual thinking. That the participants came up with a diverse list of other application scenarios further indicates the potential value of this approach, which can be worthwhile to explore.

\section{CONCLUSION}

In this work we propose a VR system to view and manipulate medical 3D images in combination with 3D printed organ models as controllers. We investigate whether the users benefit from a controller in the shape of a liver and if the size of the controller has to match the real size. In a user study with 14 medical experts we compared three different liver-shaped controllers (100\%, $75 \%$ and $50 \%$ of a real liver) and a VIVE Tracker as control condition. We tested for presence, mental workload, rotation error and conducted an interview. While the quantitative results show no significant effect for either evaluated aspect, the interviews show a preference for the $75 \%$ model. Due to the findings we can recommend (1) a high similarity of physical and virtual objects regarding shape and (2) a focus on a good manageability of the physical object depending on the task rather than simply providing an exact replica in size. The participating physicians saw a benefit using such an interaction for viewing medical data and proposed various application scenarios, which is an encouragement to do follow up studies on the size and especially weight aspects, incorporating different printing technologies and design concepts.

\section{ACKNOWLEDGMENTS}

This project has received funding from the German Federal Ministry of Education and Research (BMBF) in the grant program "Gesundes Leben" (healthy living).

\section{REFERENCES}

[1] Mahdi Azmandian, Mark Hancock, Hrvoje Benko, Eyal Ofek, and Andrew D. Wilson. 2016. Haptic Retargeting: Dynamic Repurposing of Passive Haptics for Enhanced Virtual Reality Experiences. In Proceedings of the 2016 CHI Conference on Human Factors in Computing Systems (San Jose, California, USA) (CHI '16). ACM, New York, NY, USA, 1968-1979. https://doi.org/10.1145/2858036.2858226

[2] Lonni Besançon, Paul Issartel, Mehdi Ammi, and Tobias Isenberg. 2017. Mouse, Tactile, and Tangible Input for 3D Manipulation. In Proceedings of the 2017 CHI Conference on Human Factors in Computing Systems (Denver, Colorado, USA) (CHI '17). ACM, New York, NY, USA, 4727-4740. https://doi.org/10.1145/3025453. 3025863

[3] Lonni Besançon, Anders Ynnerman, Daniel F. Keefe, Lingyun Yu, and Tobias Isenberg. 2021. The State of the Art of Spatial Interfaces for 3D Visualization. Computer Graphics Forum n/a, n/a (2021). https://doi.org/10.1111/cgf.14189 arXiv:aaaa

[4] Andy Cockburn and Bruce McKenzie. 2002. Evaluating the Effectiveness of Spatial Memory in 2D and 3D Physical and Virtual Environments. In Proceedings of the SIGCHI Conference on Human Factors in Computing Systems (Minneapolis, Minnesota, USA) (CHI '02). ACM, New York, NY, USA, 203-210. https://doi.org/ $10.1145 / 503376.503413$

[5] Balázs Faludi, Esther I Zoller, Nicolas Gerig, Azhar Zam, Georg Rauter, and Philippe C Cattin. 2019. Direct Visual and Haptic Volume Rendering of Medical Data Sets for an Immersive Exploration in Virtual Reality. In International Conference on Medical Image Computing and Computer-Assisted Intervention. Springer, Springer, Cham, na, 29-37. 
[6] Chihua Fang, Jihyun An, Antonio Bruno, Xiujun Cai, Jia Fan, Jiro Fujimoto, Rita Golfieri, Xishan Hao, Hongchi Jiang, Long R Jiao, et al. 2020. Consensus recommendations of three-dimensional visualization for diagnosis and management of liver diseases. Hepatology International 14, 4 (2020), 437-453.

[7] Sandra G Hart and Lowell E Staveland. 1988. Development of NASA-TLX (Task Load Index): Results of empirical and theoretical research. Advances in psychology 52 (1988), 139-183.

[8] Ken Hinckley, Randy Pausch, John C. Goble, and Neal F. Kassell. 1994. Passive Real-world Interface Props for Neurosurgical Visualization. In Conference Companion on Human Factors in Computing Systems (Boston, Massachusetts, USA) (CHI '94). ACM, New York, NY, USA, 232-. https://doi.org/10.1145/259963.260443

[9] Hiroshi Ishii and Brygg Ullmer. 1997. Tangible Bits: Towards Seamless Interfaces Between People, Bits and Atoms. In Proceedings of the ACM SIGCHI Conference on Human Factors in Computing Systems (Atlanta, Georgia, USA) (CHI '97). ACM New York, NY, USA, 234-241. https://doi.org/10.1145/258549.258715

[10] Robert J.K. Jacob, Audrey Girouard, Leanne M. Hirshfield, Michael S. Horn, Orit Shaer, Erin Treacy Solovey, and Jamie Zigelbaum. 2008. Reality-based Interaction: A Framework for post-WIMP Interfaces. In Proceedings of the SIGCHI Conference on Human Factors in Computing Systems (Florence, Italy) (CHI '08). ACM, New York, NY, USA, 201-210. https://doi.org/10.1145/1357054.1357089

[11] James H Johnson. 2002. Importance of dissection in learning anatomy: personal dissection versus peer teaching. Clinical Anatomy 15, 1 (2002), 38-44.

[12] Andrey Krekhov, Katharina Emmerich, Philipp Bergmann, Sebastian Cmentowski, and Jens Krüger. 2017. Self-Transforming Controllers for Virtual Reality First Person Shooters. In Proceedings of the Annual Symposium on Computer-Human Interaction in Play (Amsterdam, The Netherlands) (CHI PLAY '17). ACM, New York, NY, USA, 517-529. https://doi.org/10.1145/3116595.3116615

[13] Eun Kwon, Gerard J. Kim, and Sangyoon Lee. 2009. Effects of Sizes and Shapes of Props in Tangible Augmented Reality. In Proceedings of the 2009 8th IEEE International Symposium on Mixed and Augmented Reality (ISMAR '09). IEEE Computer Society, Washington, DC, USA, 201-202. https://doi.org/10.1109/ ISMAR.2009.5336463

[14] Irene López García and Eva Hornecker. 2021. Scaling Data Physicalization How Does Size Influence Experience?. In Proceedings of the Fifteenth International Conference on Tangible, Embedded, and Embodied Interaction (Salzburg, Austria) (TEI '21). Association for Computing Machinery, New York, NY, USA, Article 8, 14 pages. https://doi.org/10.1145/3430524.3440627

[15] Alessandro Marro, Taha Bandukwala, and Walter Mak. 2016. Three-dimensional printing and medical imaging: a review of the methods and applications. Current problems in diagnostic radiology 45, 1 (2016), 2-9.

[16] Nicolas Martelli, Carole Serrano, Hèlène van den Brink, Judith Pineau, Patrice Prognon, Isabelle Borget, and Salma El Batti. 2016. Advantages and disadvantages of 3-dimensional printing in surgery: Asystematic review. Surgery 159, 6 (2016), $1485-1500$.

[17] Thierry Morineau, Xavier Morandi, Nadège Le Moëllic, Sylma Diabira, Laurent Riffaud, Claire Haegelen, Pierre-Louis Hénaux, and Pierre Jannin. 2009. Decision Making During Preoperative Surgical Planning. Human Factors 51, 1 (2009),
67-77.

[18] Thomas Muender, Anke V. Reinschluessel, Sean Drewes, Dirk Wenig, Tanja Döring, and Rainer Malaka. 2019. Does It Feel Real?: Using Tangibles with Different Fidelities to Build and Explore Scenes in Virtual Reality. In Proceedings of the 2019 CHI Conference on Human Factors in Computing Systems (Glasgow, Scotland Uk) (CHI '19). ACM, New York, NY, USA, Article 673, 12 pages. https: //doi.org/10.1145/3290605.3300903

[19] A. Reinschluessel, R. Fischer, C. Schumann, V. Uslar, T. Muender, U. Katzky, H. Kißner, V. Kraft, M. Lampe, T. Lück, K. Bock-Müller, H. Nopper, S. Pelzl, D. Wenig, A. Schenk, D. Weyhe, G. Zachmann, and R. Malaka. 2019. Introducing Virtual \& 3D-Printed Models for Improved Collaboration in Surgery. In Proceedings of the 18. Annual Meeting of the German Society of Computer-and Robot-Assisted Surgery,, Oliver Burgert (Ed.), Vol. 18. Deutsche Gesellschaft für die Computerund Roboterassistierte Chirurgie e.V., Stuttgart, Germany, 253-258.

[20] Anke V. Reinschluessel, Thomas Muender, Verena Uslar, Dirk Weyhe, Andrea Schenk, and Rainer Malaka. 2019. Tangible Organs: Introducing 3D Printed Organ Models with VR to Interact with Medical 3D Models. In Extended Abstracts of the 2019 CHI Conference on Human Factors in Computing Systems (Glasgow, Scotland Uk) (CHI EA '19). ACM, New York, NY, USA, Article LBW1816, 6 pages. https://doi.org/10.1145/3290607.3313029

[21] B. Reitinger, A. Bornik, R. Beichel, and D. Schmalstieg. 2006. Liver Surgery Planning Using Virtual Reality. IEEE Computer Graphics and Applications 26, 6 (11 2006), 36-47.

[22] A. Schenk, S. Zidowitz, H. Bourquain, M. Hindennach, C. Hansen, H.K. Hahn, and H.-O. Peitgen. 2008. Clinical relevance of model based computer-assisted diagnosis and therapy, In Medical Imaging 2008: Computer-Aided Diagnosis. Progress in Biomedical Optics and Imaging - Proceedings of SPIE 6915, 691502-1691502-19. https://doi.org/10.1117/12.780270

[23] Adalberto L. Simeone, Eduardo Velloso, and Hans Gellersen. 2015. Substitutiona Reality: Using the Physical Environment to Design Virtual Reality Experiences. In Proceedings of the 33rd Annual ACM Conference on Human Factors in Computing Systems (Seoul, Republic of Korea) (CHI'15). ACM, New York, NY, USA, 3307-3316. https://doi.org/10.1145/2702123.2702389

[24] C Lee Ventola. 2014. Medical applications for 3D printing: current and projected uses. Pharmacy and Therapeutics 39, 10 (2014), 704.

[25] Colin Ware and Jeff Rose. 1999. Rotating virtual objects with real handles. ACM Transactions on Computer-Human Interaction (TOCHI) 6, 2 (1999), 162-180.

[26] Bob G Witmer and Michael J Singer. 1998. Measuring presence in virtual environments: A presence questionnaire. Presence 7, 3 (1998), 225-240.

[27] Yingda Xia, Lingxi Xie, Fengze Liu, Zhuotun Zhu, Elliot K Fishman, and Alan L Yuille. 2018. Bridging the gap between $2 \mathrm{~d}$ and $3 \mathrm{~d}$ organ segmentation with volumetric fusion net. In International Conference on Medical Image Computing and Computer-Assisted Intervention. Springer, Springer, Cham, na, 445-453.

[28] Jiawen Yao, Jinzheng Cai, Dong Yang, Daguang Xu, and Junzhou Huang. 2019. Integrating 3D Geometry of Organ for Improving Medical Image Segmentation. In International Conference on Medical Image Computing and Computer-Assisted Intervention. Springer, Springer, Cham, na, 318-326. 